\title{
Ein grosser Erfahrungsschatz
}

Hansjakob Müller

* Bezug über: g.alsmeier@ hippel-lindau.de oder: hansjakob.mueller@unibas.ch

Korrespondenz:

Prof. Dr. med. Hansjakob Müller Abt. Medizinische Genetik UKBB/DBM

Universität Basel

Römergasse 8

CH-4005 Basel

hansjakob.mueller@unibas.ch

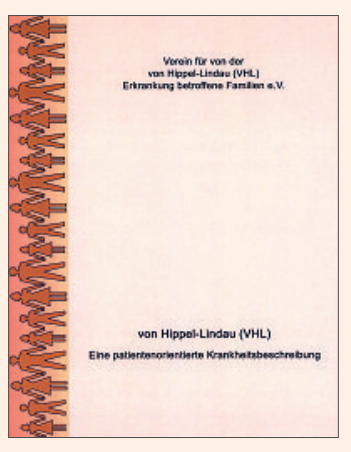

VHL-Verein und Hartmut P.H. Neumann (Hrsg.) von Hippel-Lindau (VHL). Eine patientenorientierte Krankheitsbeschreibung

Norderstedt:

Books on Demand $\mathrm{GmbH}$; 2010. 135 Seiten. Gratis. ISBN 978-3-8391-7042-7*

Die von Hippel-Lindau-Erkrankung (VHL) ist eine sehr seltene, jedoch für die Betroffenen sehr schwere Erbkrankheit. Vielfach hört man höchstens im Studium davon; in Erinnerung bleibt meist einzig der Name. Sobald man jedoch mit der Diagnostik der VHL-Erkrankung sowie der Betreuung von Patienten und ihren Angehörigen konfrontiert ist, wird VHL zu einer grossen ärztlichen Herausforderung.

Die autosomal-dominant vererbte VHL-Veranlagung führt zur Entstehung von Zysten und vor allem Tumoren in verschiedenen Organen, so $\mathrm{zu}$ Hämangioblastomen im Auge, im Gehirn oder entlang des Rückenmarkes, zu Tumoren des Endolymphsacks im Innenohr, zu klarzelligen Nierenkarzinomen, zu Inselzelltumoren im Pankreas, aber auch zu Zellwucherungen im Nebenhoden oder den breiten Mutterbändern sowie zu Phäochromozytomen. Die ersten Krankheitssymptome sind häufig wenig spektakulär: Kopfschmerzen bei Hämangioblastomen der hinteren Schädelgrube oder erhöhter Blutdruck bei einem Phäochromozytom. Nierenzysten und -karzinome bleiben vorerst gerne stumm. Die auch intrafamiliär sehr variablen Symptome werden durch die in den verschiedenen Organen möglichen Tumoren ausgelöst. Häufig braucht es daher Zeit, bis die einschlägige klinische Verdachtsdiagnose gestellt wird, die durch eine sorgfältig erhobene Familienanamnese bei $80 \%$ der Patientinnen und Patienten erhärtet und durch einen Gentest zuverlässig verifiziert werden kann. Bei etwa 20\% der Betroffenen liegt eine Neumutation des VHL-Gens vor.

Die ärztliche Betreuung der Patienten mit dieser Multiorgankrankheit macht ein konzertiertes Zusammenwirken vieler medizinischer Spezialdisziplinen notwendig. Dies kann für die Betroffenen leicht zu einer frustrierenden Odyssee führen: Man reicht sie ohne eigentlichen Lotsendienst von Spezialist zu Spezialist weiter. Sie müssen ihre Krankengeschichte unerfahrenen Assistenzärztinnen und -ärzten immer wieder von neuem erzählen und werden mit unter- schiedlichen Meinungen über Art und Rhythmus der Kontrolluntersuchungen und Therapiemassnahmen konfrontiert. Eine Verbesserung dieser Situation drängt sich vielerorts auch in der Schweiz auf.

Der deutsche «Verein für von der von HippelLindau (VHL) Erkrankung betroffene Familien e.V.» hat eben eine aktualisierte Version ihres erstmals 2002 publizierten Leitfadens über die Diagnostik und Therapie der VHL herausgegeben. Sie stellt diesen auch schweizerischen Ärztinnen und Ärzten, die sich um Patienten mit VHL kümmern, unentgeltlich zur Verfügung. Dieser Leitfaden beruht auf dem grossen Erfahrungsschatz des Freiburger Pioniers der Erforschung der VHL, Professor Dr. Hartmut P. H. Neumann. Aber auch andere deutsche Fachleute, die Erfahrungen im Umgang mit VHL-Patienten sammeln konnten, kommen zu Wort.

In den einzelnen Kapiteln wird auf die Manifestation von VHL in den verschiedenen gefährdeten Organen, auf die molekulargenetische Diagnostik der VHL-Veranlagung, auf Kontrolluntersuchungen, speziell auch im Kindes- und Jugendlichenalter, auf moderne diagnostische Verfahren und Therapiemöglichkeiten sowie auf die Hilfe bei der Krankheitsbewältigung und Lebensplanung eingegangen. Die Erstbeschreiber der Erbkrankheit werden vorgestellt, ferner soziale Aspekte (Versicherungsschutz usw.) behandelt. Es bleibt einzig zu ergänzen, dass 2004 auch in der Schweiz eine tatkräftige Selbsthilfegruppe gegründet wurde (www.switzer land.vhl-europa.org).

Der Text des Leitfadens ist praxisorientiert und gut verständlich abgefasst. Daher eignet er sich auch als Informationsquelle für Laien. Besonders nützlich dürfte er sich bei der fachübergreifenden ärztlichen Zusammenarbeit erweisen.

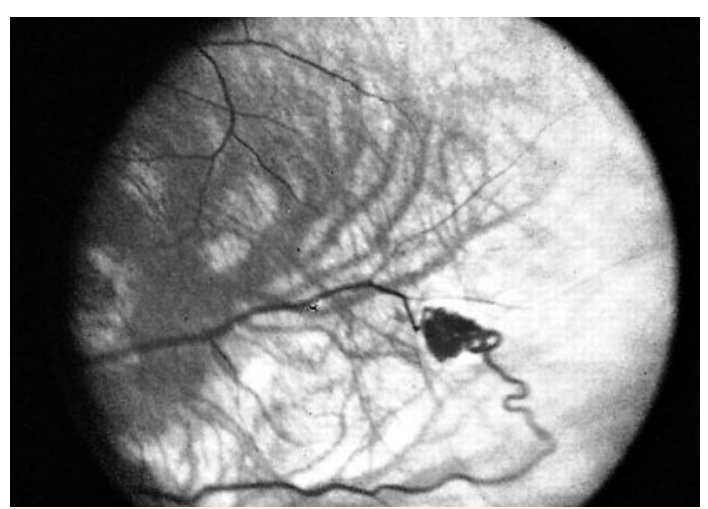

Hämangioblastom der Netzhautgefässe, verursacht durch die von Hippel-Lindau-Erkrankung. 